\title{
Quality evaluation of Dendrobium based on ultra-performance liquid chromatography (UPLC) and chemometrics
}

\author{
Caimei Gu, Xiang Zhang, Labin Wu, Xue Jiang, Linfang Huang* \\ Institute of Medicinal Plant Development, Chinese Academy of Medical Sciences and Peking Union Medical College, Beijing 100193, China.
}

\begin{tabular}{|c|c|}
\hline ARTICLE INFO & ABSTRACT \\
\hline $\begin{array}{l}\text { Article history: } \\
\text { Received on: } 08 / 11 / 2016 \\
\text { Revised on: } 27 / 11 / 2016 \\
\text { Accepted on: } 10 / 12 / 2016 \\
\text { Available online: } 31 / 01 / 2017\end{array}$ & $\begin{array}{l}\text { Dendrobium, is one of the most important herbs in the traditional Chinese medicine, which widely distributed } \\
\text { throughout Asia, Europe and Australia. Nowadays, the quality and safety of different Dendrobium species in } \\
\text { usage are still big problems, and few studies on it. In this study, } 15 \text { important species ( } 60 \text { samples) of } \\
\text { Dendrobium were studied based on the compounds, including total polysaccharides, total alkaloids, dendrobine } \\
\text { and bibenzyl by ultra-performance liquid chromatography. The results showed that } D \text {. devonianum has the }\end{array}$ \\
\hline $\begin{array}{l}\text { Key words: Dendrobium; } \\
\text { polysaccharides; alkaloids; } \\
\text { ultra-performance liquid } \\
\text { chromatography; } \\
\text { chemometrics. }\end{array}$ & $\begin{array}{l}\text { chrysotoxum. This investigation provides scientific evidences for quality control and sustained development of } \\
\text { Dendrobium plants. }\end{array}$ \\
\hline
\end{tabular}

\section{INTRODUCTION}

Dendrobium (Shihu), a well-known traditional Chinese medicine (TCM) and healthy food with widely using for 3000 years, is regarded as a top-grade drug according to Shen Nong's Herbal Classic. Dendrobium presents benefits to the stomach by producing biofluids, which enhances its Yin function and eliminates malignant heat from the human body. It is used to treat disorders such as asthenia, dim vision and retching. Besides, Dendrobium has been developed as a superior functional tea for eliminating lung heat and invigorating the spleen in ancient China (Li, 2004). Shihu widely distributed throughout Asia, Europe and Australia, with more than 1100 species. About 74 species and 2 variations of Dendrobium plants were found in China, and about 30 species are used in TCM (Pharmacopoeia Commission of Prc, 2010). Four specific Dendrobium species were recorded in the Pharmacopoeia of the People's Republic of

* Corresponding Author

Linfang Huang (Email: lfhuang @ implad.ac.cn)
China (PPRC, 2010 version), including D. officinale, D. nobile, D. fimbriatum and D. chrysotoxum. The latter three species are called Shihu in PPRC, and D. officinale is called Tiepi Shihu. Nowadays, numerous Dendrobium species exist in medicinal markets in China, including $D$. officinale, $D$. nobile, D. fimbriatum, $D$. chrysotoxum, D. chryseum, D. thyrsiflorum, D. chrysanthum, D. loddigesii, D. devonianum, D. moniliforme, etc. (Fig. 1) Recent studies have showed the big differences of chemical composition between different main origins in the same species ( $\mathrm{Li}$ et al., 2013). However, due to multiple origins, their active compounds including polysaccharides, alkaloids, dendrobine and bibenzyl were greatly varied. Although there exists the quality differences between species (Bao et al., 2005), few researches have reported the differences between different species. Therefore, it is urgent to evaluate these species to ensure the safety in usage. The present work was carried out to scientifically compare the active compounds of Dendrobium from different orgins. We collected 15 species (60 samples) of Dendrobium from different locations in this investigation, which are the main commercial species in China. 
We investigated the contents of bioactive compounds, including total polysaccharides, total alkaloids, dendrobine and bibenzyls, to evaluate the quality of Dendrobium by ultravioletvisible spectrophotometry (UV-VIS), gas chromatography (GC), UPLC (Xu et al., 2005; Huang and Li, 2007; Lu et al., 2010; Xia et al., 2008), and other technologies (Deng et al., 2016; Chen et al., 2016; Jin et al., 2016). The results of this investigation are significant for scientific cultivation of Dendrobium with the purpose of sustained development of resources in China.
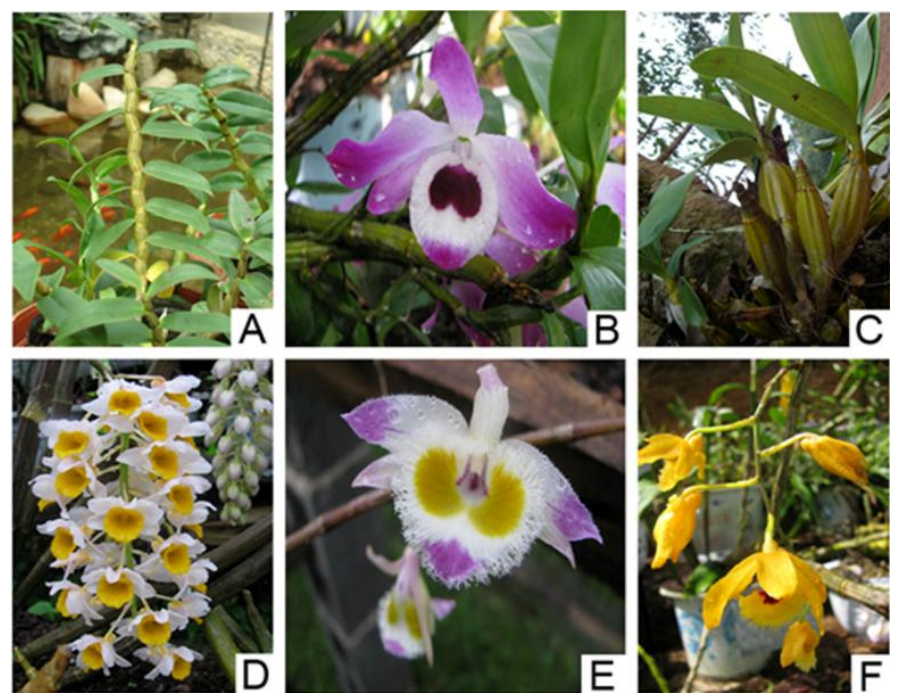

Fig. 1: The major Dendrobium of different species. (A: D. officinale; B:D. nobile; C: D. chrysotoxum; D: D. thyrsiflorum; E: D. devonianum; F: D. fimbriatum)

\section{MATERIALS AND METHODS}

\section{Chemicals, reagents and materials}

Waters Acquity ${ }^{\mathrm{TM}}$ UPLC-diode array detector (UPLC-

DAD) (Waters Co., Milford, MA, USA); Mettler AB135-S electronic analytical balance (Mettler-Toledo., Greifensee, Zurich, Switzerland); electric thermostatic water bath (Beijing Analyst Co., Beijing, China); KQ-400KDB ultrasonic cleaner (Kunshan Co., Hangzhou, China).

Methanol and acetonitrile (chromatographic grade, Fisher Co., Fairlawn, NJ, USA); ultrapure water (Milli-Q system, 18.2 M $\Omega$, Millipore, Bedford, MA, USA); sulfuric acid (98\%), redistilled phenol, potassium hydrogen phthalate (KHPh), formic acid, methanol, $\mathrm{NaOH}$, bromocresol green, absolute ethyl alcohol, petroleum ether and chloroform (analytic grade, Beijing Chemical Co., Beijing, China); dendrobine standard (>98\%, Chengdu Beth Reagent Co., Sichuan, China); scoparone, gigantol, erianin and Danhydrous dextrose standards (>98\%, National Institutes for Food and Drug Control, Beijing, China).

All of the medicinal and plant materials were identified by Prof. Lin Yulin (Institute of Medicinal Plant Development, Chinese Academy of Medical Sciences and Peking Union Medical College, Beijing, China) and deposited at the Herbarium of
Institute of Medicinal Plant Development, Chinese Academy of Medical Sciences (Beijing, China) with $4{ }^{\circ} \mathrm{C}$ storage.

\section{Determination of total polysaccharides and alkaloids in Dendrobium}

Fresh stems of Dendrobium were processed at $105{ }^{\circ} \mathrm{C}$ for $1 \mathrm{~h}$ to inhibit enzymes in the samples. Then, samples were dried at $60{ }^{\circ} \mathrm{C}$, and were pulverized (through 60-mesh sieve). Extracted with alkaline chloroform, the total alkaloids were determined with the bromocresol green colorimetry. The polysaccharides were extracted with $80 \%$ ethanol after the impurity was eliminated with petroleum ether, and determined with phenol-concentrated sulfuric acid colorimetry (Lu et al., 2010).

\section{Determination of bibenzyl in Dendrobium by UPLC}

About $0.25 \mathrm{~g}$ of the sample was weighted accurately and extracted with $50 \mathrm{~mL} 80 \%$ methanol. The samples were extracted in an ultrasonic bath for $45 \mathrm{~min}$; and were filtered through a $0.22 \mu \mathrm{m}$ membrane, then were analyzed by UPLC.

Chromatographic analysis was performed using a $\mathrm{BEH}$ $\mathrm{C}_{18}$ column $(100 \mathrm{~mm} \times 2.1 \mathrm{~mm}, 1.7 \mu \mathrm{m}$, Waters $)$ at $230 \mathrm{~nm}$ by UPLC. The binary gradient elution system consisted of acetonitrile (A) and water (0.005\% formic acid) (B); and separation was achieved using the following gradient: $0 \mathrm{~min}$ to $7 \mathrm{~min}, 5 \%$ to $40 \%$ A; $7 \mathrm{~min}$ to $10 \mathrm{~min}, 40 \%$ to $60 \% \mathrm{~A} ; 10 \mathrm{~min}$ to $14 \mathrm{~min}, 60 \%$ to $100 \% \mathrm{~A}$. The flow rate was $0.25 \mathrm{~mL} \cdot \mathrm{min}^{-1}$; and the column and sample temperatures were maintained at $25^{\circ} \mathrm{C}$ and $15^{\circ} \mathrm{C}$, respectively. Scoparone, gigantol, and erianin standard solutions were prepared with methanol and stored at $4{ }^{\circ} \mathrm{C}$.

\section{Methodological evaluation}

The calibration curve, stability, precision, repeatability and recovery rate were performed to validate the above methods (Table 1). Linearity was examined using the standard solutions by calibration curves test. The calibration curves were constructed by plotting the peak area $(\mathrm{Y})$ versus the concentrations $(\mathrm{X})$ of the standard solutions. All calibration curves showed good linear regressions and were within the test ranges. The precision was determined by intra-day and inter-day variations through the analysis of the six replicate samples within one day and three consecutive days, respectively. Six independently prepared solutions from the same sample were analyzed for repeatability testing. In the determination of total polysaccharides and alkaloids, the stability of the samples was analyzed at $0,10,20,40,60,80$, 100 and $120 \mathrm{~min}$ in $4{ }^{\circ} \mathrm{C}$ storage, respectively. The results present that this experiment was stable within $2 \mathrm{~h}$ with $\mathrm{RSD}<1.29 \%$. In the determination of dendrobine and bibenzyl, the stability of the samples was analyzed at $0,2,4,8,12,24$, and $36 \mathrm{~h}$ with $4{ }^{\circ} \mathrm{C}$ storage, respectively. The sample solutions were stable within $36 \mathrm{~h}$ with $\mathrm{RSD}<3.64 \%$. The average recoveries of the four compounds ranged from $93.72 \%$ to $99.07 \%$ ( $\mathrm{RSD}<2.63 \%$ ). These validation results indicated that the methods used in the quantitative determination of the target compounds were scientific. 
Table 1: Methodological validation.

\begin{tabular}{|c|c|c|c|c|c|c|c|c|}
\hline \multirow{2}{*}{$\begin{array}{l}\text { Standard } \\
\text { compound }\end{array}$} & \multirow{2}{*}{ Regression equation } & \multirow{2}{*}{$\begin{array}{c}\text { Correlation } \\
\text { coefficient } \\
\left(R^{2} N=6\right)\end{array}$} & \multirow{2}{*}{ Linear range } & \multirow{2}{*}{$\begin{array}{c}\text { Stability } \\
\text { (RSD\%) } \\
\mathrm{N}=5\end{array}$} & \multirow{2}{*}{$\begin{array}{c}\text { Precision } \\
\text { (RSD\%) } \\
\text { N=5 }\end{array}$} & \multirow{2}{*}{$\begin{array}{c}\text { Repeat- } \\
\text { ability } \\
\text { (RSD\%) } \\
\text { N=5 }\end{array}$} & \multicolumn{2}{|c|}{$\operatorname{Recovery}(\mathbf{N}=5)$} \\
\hline & & & & & & & $\operatorname{Mean}(\%)$ & $\operatorname{RSD}(\%)$ \\
\hline D-anhydrous dextrose & $Y=0.0746 x+0.0061$ & 0.9994 & $0.01-0.06 \mathrm{mg} \cdot \mathrm{mL}^{-1}$ & 0.126 & 2.38 & 3.93 & 99.07 & 1.23 \\
\hline Akaloid & $Y=14.568 x+0.4735$ & 0.9991 & $1.13-5.65 \mu \mathrm{g} \cdot \mathrm{mL}^{-1}$ & 1.29 & 2.38 & 3.93 & 98.16 & 2.63 \\
\hline Dendrobine & $Y=76414 x-86.49$ & 0.9997 & $0.05-0.3 \mathrm{mg}$ & 1.63 & 1.32 & 1.85 & 96.47 & 1.83 \\
\hline Scoparone & $Y=46553 x-12997$ & 0.9998 & $2.0-350.0 \mathrm{ng}$ & 3.64 & 2.37 & 2.58 & 94.44 & 1.28 \\
\hline Gigantol & $Y=42664 x-19172$ & 0.9998 & $5.0-285.0 \mathrm{ng}$ & 3.25 & 2.29 & 1.52 & 93.72 & 2.46 \\
\hline Erianin & $Y=26366 x-103254$ & 0.9997 & $5.0-290.0 \mathrm{ng}$ & 2.86 & 1.60 & 2.33 & 96.35 & 1.39 \\
\hline
\end{tabular}

Table 2: Environmental factors analysis based on Pearson correlation.

\begin{tabular}{|c|c|c|c|c|c|c|c|c|c|c|c|c|}
\hline \multirow[t]{2}{*}{ Species } & \multirow[t]{2}{*}{ compounds } & \multicolumn{11}{|c|}{ Environmental factors } \\
\hline & & $X_{1}$ & $X_{2}$ & $X_{3}$ & $X_{4}$ & $X_{5}$ & $X_{6}$ & $X_{7}$ & $X_{8}$ & $X_{9}$ & $X_{10}$ & $X_{11}$ \\
\hline D. officinale & Polysaccharides & 0.066 & 0.200 & 0.085 & 0.099 & 0.326 & -0.120 & -0.291 & -0.028 & -0.035 & 0.071 & 0.150 \\
\hline \multirow[t]{2}{*}{ D. nobile } & Alkaloids & -0.001 & -0.023 & 0.049 & 0.058 & -0.098 & 0.344 & -0.195 & 0.115 & 0.095 & 0.095 & -0.013 \\
\hline & Dendrobine & -0.275 & -0.468 & -0.077 & 0.347 & $-0.512^{b}$ & -0.013 & 0.127 & 0.128 & 0.140 & -0.201 & -0.489 \\
\hline \multirow[t]{2}{*}{ D. chrysotoxum } & Gigantol & 0.153 & 0.235 & 0.088 & 0.230 & 0.193 & 0.035 & -0.273 & -0.110 & -0.136 & 0.117 & 0.217 \\
\hline & Erianin & $0.820^{\mathrm{a}}$ & $0.703^{\mathrm{b}}$ & -0.575 & 0.218 & -0.040 & 0.387 & -0.231 & $0.704^{b}$ & 0.658 & $0.732^{b}$ & $0.683^{b}$ \\
\hline
\end{tabular}

a Correlation is significant at the 0.01 level (2-tailed); b Correlation is significant at the 0.05 level (2-tailed); $X_{1}$, Active accumulative temperature; $X_{2}$, Average annual air temperature; $X_{3}$, Elevation; $X_{4}$, Relative humidity; $X_{5}$, Annual sunshine time; $X_{6}$, Annual precipitation; $X_{7}$, Soil; $X_{8}$, Maximum air temperature in July; $X_{9}$, Average air temperatur in July; $X_{10}$, Minimumair temperature in January; $X_{11}$, Average air temperature in January.

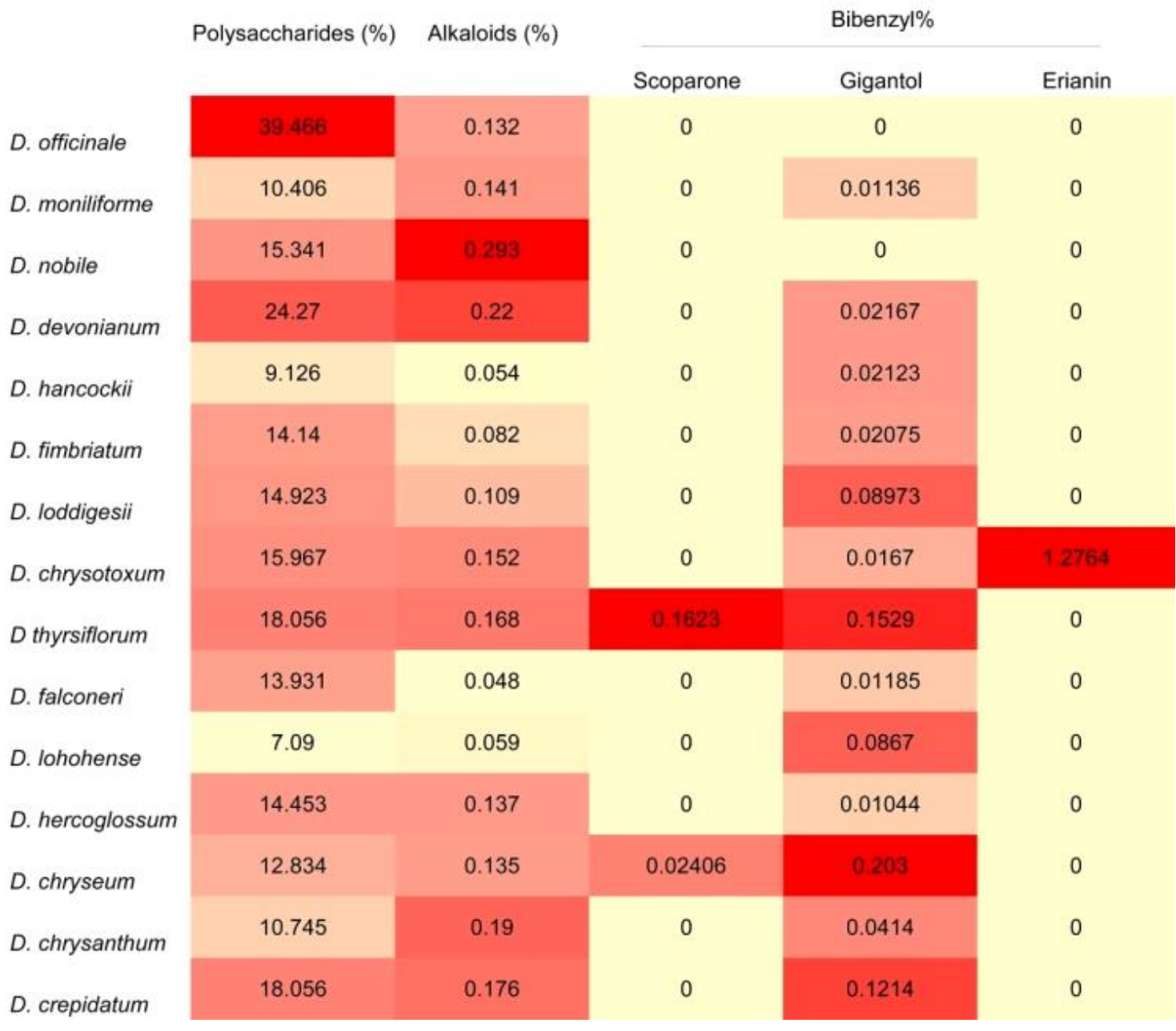

Fig. 2: Contents of total polysaccharides, alkaloids and bibenzyl in different species of Dendrobium. 


\section{RESULTS AND DISCUSSION}

\section{Determination and analysis of total polysaccharides and alkaloids in Dendrobium}

The contents of total polysaccharide and alkaloid in 15 species of Dendrobium are presented in Fig. 2. The results show that $D$. officinale has the highest content of total polysaccharide among all of the species; and D. nobile has the highest content of total alkaloids among all the species, while, D. devonianum has the secondary top contents both in polysaccharides and alkaloids.
Polysaccharides are one of the most important compounds in TCM, and the content of polysaccharide is the main criterion in evaluating the quality of $D$. officinale in the PPRC (Luo et al., 2000; Huang et al., 1996). Luo et al. has separated four polysaccharides with higher scavenging activity of hydroxyl radical and DPPH radical from $D$. nobile and $D$. denneanum (Luo et al., 2010; Fan et al., 2010; Luo et al., 2009; Huang et al., 2016). Wang, et al. has separated nine polysaccharides with higher antitumor activities against Sarcoma 180 in vivo and HL-60 in vitro (Wang et al., 2009).
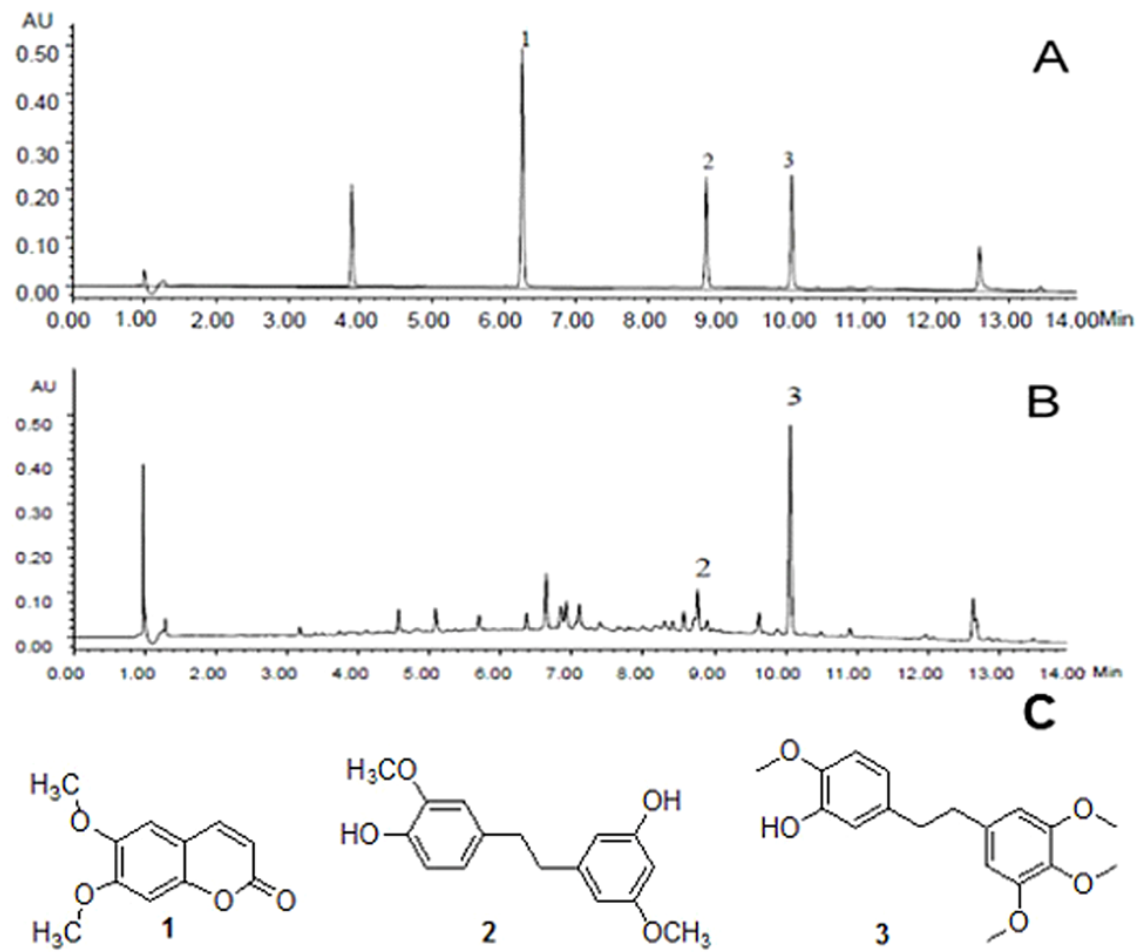

Fig. 3: UPLC chromatography and the structure of three compounds (A. UPLC chromatography of standards. B. UPLC chromatography of D. Chrysotoxum. C. The structure of three compounds, 1. Scoparone; 2. Giganto; 3. Erianin.)

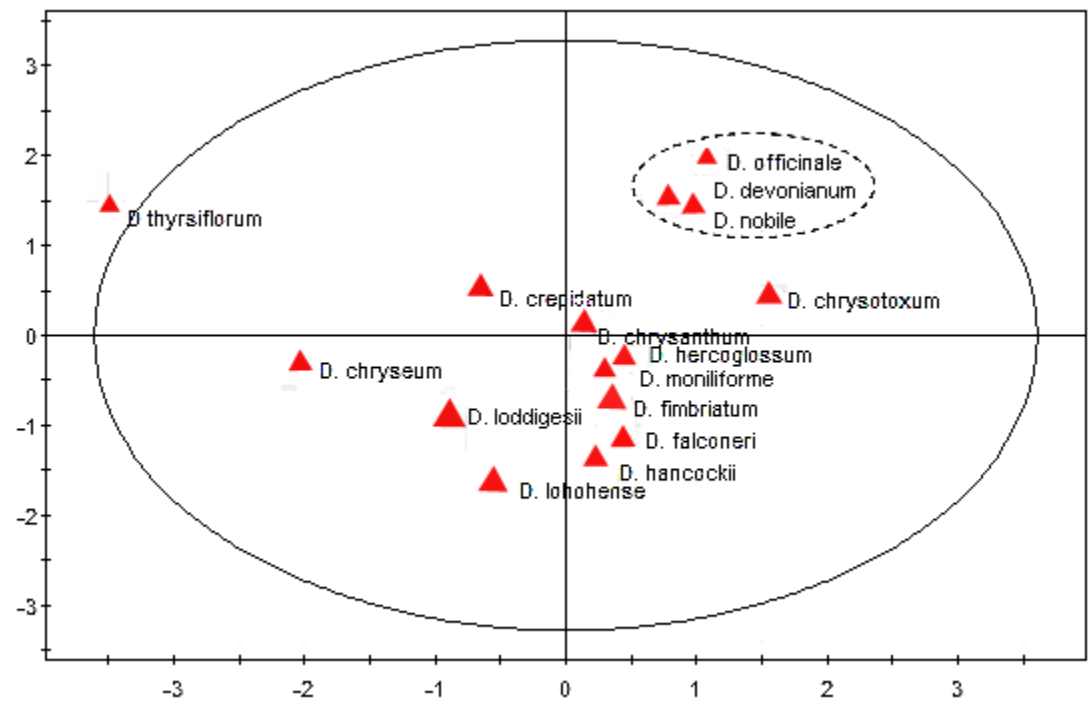

Fig. 4: Scatter diagram of Dendrobium species based on polysaccharides and alkaloids. 
Zhao et al. have got a polysaccharides, named as DCLP, from D. chrysotoxum. And they believe that DCLP has antioxidative, hypoglycemic and immune stimulating effects in vitro and in vivo (Zhao et al., 2007). Zha, et al. showed that polysaccharides of HPS-1B23, which was separated from $D$. huoshanense possessed potent stimulating functions on IFN- $\gamma$ and TNF- $\alpha$ production in the culture medium of splenocytes and macrophages, respectively (Zha et al., 2007). Due to the complexity of these polysaccharides in Dendrobium, measuring the total polysaccharides in combined with chemometrics becoming an effective way for evaluating qualities comprehensively.

Based on the content of polysaccharides, it indicates that $D$. officinale and $D$. devonianum have the higher qualities compared to other species. And it is the reason that why $D$. officinale is recorded as an official Dendrobium.

Alkaloids are other important compounds in Dendrobium. The alkaloids from Dendrobium presented neuroprotective effects on rat cortical neurons injured by oxygenglucose deprivation and reperfusion (Tong et al., 2016; He et al., 2016; Wang et al., 2010). Besides, its alkaloids have inhibitory effects on memory impairment (Li et al., 2010). And alkaloids from $D$. nobile have the bioactivity of prevention on diabetic cataract in rat lens (Wei and Long, 2008).

In the determination of alkaloids, it shows that D. nobile has the highest content. This result indicates that $D$. officinale and D. nobile have the different pharmacological values.

\section{PCA of total polysaccharides, alkaloids and bibenzyl in Dendrobium}

PCA uses an $\mathrm{N}$-dimensional vector approach to separate samples on the basis of the cumulative correlation of all metabolite data and then identifies the vector (eigenvector) that yields the greatest separation among samples without requiring prior knowledge of the data sets.

The contents of total polysaccharides, alkaloids in different species of Dendrobium were analyzed by PCA. As shown in Fig. 4, D. officinale, D. devonianum and D. nobile cluster together. It indicates the higher similarities among these three major species. Both $D$. officinale and $D$. nobile have been recorded in China Pharmacopoeia except $D$. devonianum.

This species is widely used in southwest China (Ding, 2004; Cui et al., 2004), but has not attracted attentions in academic. Fig. 2 also presents that $D$. devonianum has the secondary top contents of polysaccharides and alkaloids among all the species. This result confirms the usage value of $D$. devonianum in folk. It also indicates that $D$. devonianum, $D$. officinale and $D$. nobile have significant differences with other species based on chemical components. Besides, they have genetic relationship based on morphology. In taxonomy, these three species belong to Sect Dendrobium (Flora of China, 1999). Therefore, it is suggested that $D$. devonianum could be recorded as an official Dendrobium in pharmacopoeia of China to alleviate stressing Dendrobium resources in markets.

\section{Determination and analysis of bibenzyl in Dendrobium by UPLC}

Experiments were conducted to optimize the sample preparation and chromatographic conditions. UPLC chromatographic conditions were developed to consider factors such as mobile phase, modifier and flow rate. With the optimal conditions, the analyzed peaks were well separated from each other. This newly explored chromatographic condition allows the simultaneous quantitative determination of 3 bibenzyl (scoparone, gigantol and erianin) in Dendrobium. The extraction investigation demonstrates that $80 \%$ methanol and 45 min ultrasonic extraction were the optimal preparation methods. The chromatographic profiling of represented sample and standard compounds are presented in Fig. 3.

15 species of Dendrobium were tested in this section. The determination of 3 bibenzyl in species of Dendrobium is shown in Fig. 2. The experiments show that only D. chrysotoxum contains gigantol and erianin; only D. chryseum and D. thyrsiflorum contain scoparone and gigantol; the species including D. moniliforme, $D$. devonianum, D. hancockii, D. fimbriatum, D. loddigesii, D. falconeri, D. lohohense, D. hercoglossum, D. chrysanthum and $D$. crepidatum contain only gigantol; while $D$. officinale and D. nobile have no bibenzyl. Gigantol exists in all the species of Dendrobium except for D. officinale and D. nobile.

Erianin is detected only in D. chrysotoxum. $D$. chrysotoxum is a special plant among all Dendrobium species with the special compound--erianin. Therefore, the marker component of D. chrysotoxum in PPRC is erianin. Erianin can induce apoptosis in tumor cells (Li et al., 2001), thus generating increased attention recent years. Confusarin and chrysotoxene, which have anti-tumor activities (Wang et al., 2001; Ma et al., 1994), are also found in D. chrysotoxum. Therefore, with the high potential in antitumor, we suggest that D. chrysotoxum should be recorded independently in the next edition of the PPRC. This result presents that only $D$. chryseum and $D$. thyrsiflorum contain scoparone; and a significant difference was found between these two species. The content of scoparone in D. chryseum ranged from $0.13 \%$ to $0.59 \%$, whereas that in D. thyrsiflorum ranged from $0.011 \%$ to $0.027 \%$. Scoparone exhibits vasorelaxant and immunosuppressive effects (Huang and Chu, 1994). Scoparone can induce neurite outgrowth by stimulating the upstream steps of ERK, PKA, PKC and CaMK II in PC12 cells (Yang et al., 2008). Besides, D. chryseum and D. thyrsiflorum also contained gigantol, which exists in most of the species of Dendrobium. In our previous investigation, we found that there is significant difference between fingerprint profiling of these species of Dendrobium. Therefore, D. chryseum and D. thyrsiflorum have the medical values and they could be used in the market of medicinal Dendrobium.

All of the Dendrobium species had gigantol except for $D$. officinale and D. nobile. Gigantol has inhibitory abilities against the growth of human leukemia cell lines K562 and HL-60, human lung adenocarcinoma A549, human hepatoma BEL-7402, and human stomach cancer SGC-7901 (Chen et al., 2008). 13 species contain gigantol and these species could not be distinguished by 
gigantol only although there is a significant difference in different species and locations of Dendrobium. But it could be considered as the marker compound to authenticate Dendrobium except for $D$. officinale and D. nobile. The active compounds from different species or different locations of Dendrobium were first compared. The results showed that $D$. officinale and $D$. nobile were considered as important species because of the highest polysaccharide and alkaloid contents, respectively. D. devonianum contains high contents of both polysaccharides and alkaloids, as well as its production scale has expanded continuously and the potential market is huge. Therefore, D. devonianum is a promising resource and it could be recorded as an official Dendrobium and an alternative medicine for Dendrobium. D. chrysotoxum could be distinguished from 4 species recorded in PPRC for big differences with other species, including the proprietary compound-erianin, appearance and biological characteristic. Its medicinal part is fat and shaped like a drum (Gong et al., 2008), in contrast, other species' are slim, Besides, D. chrysotoxum has the compound erianin and it can be the chemical marker, while the others don't have. Indeed, our previous study also showed that the $D$. chrysotoxum is different with other species using specific PCR molecular identification (Zheng et al., 2013). Thus, we suggest that $D$. chrysotoxum should be recorded independently in the next edition of PPRC. Ecological analysis showed that sunlight hours, precipitation and temperature influence the accumulation of the main compounds in D. officinale, D. nobile, and D. chrysotoxum, respectively. This investigation provides valuable information and scientific evidences for the quality controlling, cultivation expanding, sustainable development of Dendrobium resources and variety identification.

\section{CONCLUSIONS}

D. devonianum has the secondary top contents of polysaccharides and alkaloids among all the species, which can replaced $D$. officinale in medicinal usage. Erianin is a chemical marker for identifying between different species. D. chrysotoxum could be distinguished from 4 species recorded in PPRC according to the differences in chemistry and molecular. Furthermore, we can make much effort on $D$. chrysotoxum due to the anti-cancer effect of erianin. This is helpful to protect the valuable Dendrobium resources and is the guidance of clinical reasonable use.

\section{ACKNOWLEDGMENTS}

Financial support and sponsorship: The authors are grateful to the National Natural Science Foundation of China (No. 81274013 and No. 81473315) and CAMS Innovation Fund for Medical Science (CIFMS) NO. 2016-I2M-3-015.

Conflict of Interests: There are no conflicts of interest.

\section{REFERENCES}

Li, S.Z., Compendium of Materia Medica (Bencao Gangmu). Foreign Languages Press, 2004. Beijing.
2 Pharmacopoeia Commission of PRC: Pharmacopoeia of the People's Republic of China. Chemical Industry Press 2010, Beijing.

Li, W.T., Huang, L.F., Du, J., Chen, S.L., Relationships between Dendrobium quality and ecological factors based on partial least square regression. Chinese Journal of Applied Ecology. 2013; 24: 27872792.

Bao, X.S., Shun, Q.S., Chen, L.Z., The Medicinal Plants of Dendrobium (Shi-Hu) in China. Fudan University Press, 2005. Shanghai.

Xu, J., Zhao, W.M., Qian, Z.M., Guan, J., Li, S.P., Fast determination of five components of coumarin, alkaloids and bibenzyls in Dendrobium spp using pressurized liquid extraction and ultra-performance liquid chromatography. J. Sep. Sci. 2005; 33: 1580-1586.

Huang, X.Y., Yi, Y., Gas chromatographic determination of Dendrobine in Dendrobium nobile Lindl. J. Guizhou Normal Univ, 2007; 25: 92-94.

Lu, W.Y., He, K.Q., Jiang, X.Y., Ci, Q.Z., Liao, J.H., Relationship between Market Pricing and the Content of Alkaloids, Polysaccharides of Herba Dendrobii in Different Origins. Med Plant, 2010; 1: 31-33.

Xia, K.Z., Wang, Z., Chen, L.Z., Study on the Determination of Antitumor Component Erianin in Dendrobium chrysotoxum. Chin. J. MAP, 2008; 25: 63-66.

Deng, Y., Chen, L. X., Han, B. X., et al. Qualitative and quantitative analysis of specific polysaccharides in Dendrobium huoshanense by using saccharide mapping and chromatographic methods. Journal of Pharmaceutical and Biomedical Analysis, 2016, 129: 163-171.

Chen, N. D., You, T., Li, J., et al. A comparative study of three tissue-cultured Dendrobium species and their wild correspondences by headspace gas chromatography-mass spectrometry combined with chemometric methods. Journal of Food and Drug Analysis, 2016, 24(4): 839-847.

Jin, Q., Jiao, C., Sun, S., et al. Metabolic Analysis of Medicinal Dendrobium officinale and Dendrobium huoshanense during Different Growth Years. PloS one, 2016, 11(1): e0146607.

Luo, H.L., Cai, T.Y., Chen, Q.L., Mei, C.G., Li, Y.Q., Huang, M.Q., Enhancement of Dendrobium candidum polysaccharide on killing effect of LAK cells of umbilical cord blood and peripheral blood of cancer patients in vitro. Cancer, 2000;19: 1124-1126.

Huang, M.Q., Cai, T.Y., Liu, Q.L., Effects of polysaccharides from Dendrobium officinale on white blood cells and lymph cell moving inhibition factor of mice. Nat. Prod. Res, 1996; 8: 39-41.

Luo, A.X., He, X.J., Zhou, S.D., Fan, Y.J., Luo, A.S., Purification, composition analysis and antioxidant activity of the polysaccharides from Dendrobium nobile Lindl. Carbohyd. Polym, 2010; 79: 1014-1019.

Fan, Y.J., He, X.J., Zhou, S.D., Luo, A.X., He, T., Chun, Z., Composition analysis and antioxidant activity of polysaccharide from Dendrobium denneanum. Int. J. Biol. Macromol, 2010; 45: 169-173.

Luo, A.X., He, X.J., Zhou, S.D., Fan, Y.J., He, T., Chun, Z., In vitro antioxidant activities of a water-soluble polysaccharide derived from Dendrobium nobile Lindl. Extract. Int. J. Biol. Macromol, 2009; 45:359-63.

Huang, X., Nie, S., Cai, H., et al. Study on Dendrobium Officinale O-Acetyl-Glucomannan (Dendronan): Part VII. The Immunomodulatory and Antioxidant Activity. Gums and Stabilisers for the Food Industry 18. 2016: 218-226.

Wang, J.H., Luo, J.P., Zha, X.Q., Feng, B.J., Comparison of antitumor activities of different polysaccharide fractions from the stems of Dendrobium nobile Lindl. Carbohyd. Polym, 2009; 79: 114-118.

Zhao, Y.P., Son, Y.O., Kim, S.S., Jang, Y.S., Lee, J., Antioxidant and anti-hyperglycemic activity of polysaccharide isolated from Dendrobium chrysotoxum Lindl. J. Biochem. Mol. Biol, 2007; 40: 670-677.

Zha, X.Q., Luo, J.P., Luo, S.Z., Jiang, S.T., Structure identification of a new immunostimulating polysaccharide from the stems of Dendrobium huoshanense. Carbohyd. Polym, 2007; 69: 86-93.

Tong, L., Wang, L., Zhou, X., et al. Antitumor activity of Dendrobium devonianum polysaccharides based on their immunomodulatory effects in S180 tumor-bearing mice. RSC Advances, 2016; 6(46): 40250-40257. 
He, T. B., Huang, Y. P., Yang, L., et al. Structural characterization and immunomodulating activity of polysaccharide from Dendrobium officinale. International journal of biological macromolecules, 2016, 83: 34-41.

Wang, Q., Gong, Q., Wu, Q., Shi, J., Neuroprotective effects of Dendrobium alkaloids on rat cortical neurons injured by oxygen-glucose deprivation and reperfusion. Phytomed, 2010;17: 108-115.

Li, Y.F., Li, F., Gong, Q.H., Wu, Q., Shi, J.S., Inhibitory Effects of Dendrobium Alkaloids on Memory Impairment Induced by Lipopolysaccharide in Rats. Planta Med, 2010; 77: 117-121.

Wei, X.Y., Long, Y., Study on the Prevention and the Proteomics on Diabetic Cataract in Rat Lens by the Total Alkaloids Extracted from Dendrobium nobile Lindl. Nat Prod Res Dev, 2008; 20: 617-621.

Ding, C.C., Study on Tissue Culture and Rapid Propagation Technic of Dendrobium devonianum. Trop. Agric. Sci. Technol, 2004; 27: $10-11$.

Cui, Q.H., Sun, Y.Y., Li, K., Zhou, Z.M., Yan, H., Huang, C.X., A Study on Bud Proliferation and Rapid Propagation of Rare Forest Medicine Plant of Dendrobium devonianum Paxt. Acta Agri. U. Jiangxiensis, 2004; 33: 1139-1143.

Editorial committee of flora of China: Flora of China. Science Press 1999, Beijing.

Li, Y.M., Wang, H.Y., Liu, G.Q., Erianin induces apoptosis in human leukemia HL-60 cells. Acta Pharmacol., 2001; 22: 1018-1022.

Wang, T.S., Lu, Y.M., Ma, G.X., In vitro inhibition activities of leukemia k562 cells growth by constituents from D. chrysotoxum. Nat. Prod. Res, 2001; 9: 1-3.
Ma, G.X., Xu, G.J., Xu, L.S., Inhibitory effects of Dendrobium Chrysotoxum and its constituents on the mouse HePA and ESC. J. Chin Pharm. Univ, 1994; 25: 188-189.

Huang, H.C., Chu, S.H., Vasorelaxants from Chinese herbs, emodin and scoparone, possess immunosuppressive properties. Eur. J. Pharmacol, 1994; 198: 211-213.

Yang, Y.J., Lee, H.J., Choi, D.H., Huang, H.S., Lim, S.C., Lee, M.K., Effect of scoparone on neurite outgrowth in PC12 cells. Neurosci. Lett, 2008; 440: 14-18.

Chen, Y.G., Xu, J.J., Yu, H., Qing, C., Zhang, Y.L., Wang, L.Q., Liu, Y., Wang. J.H., Cytotoxic phenolics from Bulbophyllum odoratissimum. Food Chem, 2008; 107: 169-173.

Gong, J.Y., Wang, H.X., Sun, L.N., Lin, M., Yang, S.T., Study on Biological Characteristics and Cultivation Adaption of Nine Introduction Dendrobium. Northern Horticulture, 2013; 20: 75-78.

Zheng, S.H., Huang, L.F., Chen, S.L., Specific PCR molecular identification of Dendrobium chrysotoxum. Chinese Traditional and Herbal Drugs, 2013; 44: 744-748.

\section{How to cite this article:}

Gu C, Zhang X, Wu L, Jiang X, Huang L. Quality evaluation of Dendrobium based on ultra-performance liquid chromatography (UPLC) and chemometrics. J App Pharm Sci, 2017; 7 (01): 017023. 